\title{
塔里木河中下游地区荒漠河岸林群落种间关系分析
}

\author{
尹林克 ${ }^{1}$ 李 涛 $^{2}$ \\ (1 中国科学院新疆生态与地理研究所, 乌鲁木齐 830011) (2 新疆环境保护研究所, 乌鲁木齐 830011)
}

\begin{abstract}
摘 要 采用 $2 \times 2$ 列联表, 应用 Fisher 精确检验法研究了新疆塔里木河中下游荒漠河岸林群落种间关系, 测定了 16 种植物、共 120 个种对的种间联结性。研究结果表明:1）120 个种对中有 17 个种对分别在不同的样方尺度中表 现出显著或极显著的种间联结,约占总数的 $14.2 \%$; 其中 13 个种对为正关联, 4 个种对为负关联; 2 ) 不同取样面积 对种间联结性分析的有效性有影响, 不同种对表现出种间联结的最小样方尺度不同; 3) 随着样方面积的增大, 各种 对自有不同的种间联结变化规律, 可归纳为 4 种类型; 4) 17 个具种间联结的种对以灌木-草本和草本-草本的种对居 多, 占总数的 $76.5 \%$; 主要乔木树种胡杨 (Populus euphratica) 与灌木之间、灌木和灌木之间趋向独立分布。
\end{abstract} 关键词 塔里木荒漠河岸林群落 种间联结 列联表 Fisher 精确检验

\section{INTERSPECIFIC RELATIONSHIP ANALYSIS OF DESERT RIPARIAN FOREST PLANT COMMUNITIES IN THE MIDDLE AND LOWER REACHES OF THE TARIM RIVER}

\author{
YIN Lin-Ke ${ }^{1}$ and $\mathrm{Li} \mathrm{Tao}^{2}$ \\ (1 Xinjiang Institute of Ecology and Geography, Chinese Academy of Sciences, Urumqi 830011, China) \\ (2 Xinjiang Institute of Environmental Protection, Urumqi 830011, China)
}

\begin{abstract}
Based on data obtained from field investigations, this paper aims to analyze the inter-specific relationships of desert riparian forest plant communities, assess the influence of different quadrat sizes on interspecific associations, discuss what is an appropriate quadrat size for best results, and present the relationships among populations in desert riparian forests in the middle and lower reaches of the Tarim River using Fisher's exact test. The results showed that 16 species noted in 21 sampling sites formed the principal community of the desert riparian forest. The vertical structure of the forest consisted of a tree layer, shrub layer and herb layer. It was found that 4 sampling sites had 3 layers, 10 had 2 layers and 7 had 1 layer. There were 120 species pairs formed by 16 species in 210 quadrats among which 17 species pairs showed significant and very significant inter-specific associations, accounting for $14.2 \%$ of total species pairs. Thirteen species pairs were positively correlated and four species pairs were negatively correlated. The size of the quadrat influenced the interspecific associations among species pairs, but the most suitable quadrat size varied among species and sampling sites. A quadrat from $8 \mathrm{~m} \times 8 \mathrm{~m}$ to $30 \mathrm{~m} \times 30 \mathrm{~m}$ may be the most suitable size for analysis of inter-specific associations of desert riparian forest communities in Tarim River basin. Different species pairs had different minimum quadrat sizes. There was spatial variance in the interaction between different populations of the desert riparian forest. Different patterns and relationships of inter-specific associations were found as the size of the quadrat increased. Generally, four types of changes in species associations were observed with changing quadrat size. For the first type, the correlative significance of species pairs changed from high to low and then to high as the quadrat size changed from the minimum size of $2 \mathrm{~m} \times 2 \mathrm{~m}$ to the maximum size of $32 \mathrm{~m} \times 64 \mathrm{~m}$. These species pairs accounted for $17.6 \%$ of the total species pairs. In the second type of variation, the correlative significance of species pairs changed from low to high and then to low with an increase in quadrat size. These species pairs accounted for $41.2 \%$ of the total. For the third type, the significant and very significant inter-specific associations occurred in quadrats that ranged in size from $8 \mathrm{~m} \times 16 \mathrm{~m}$ to $32 \mathrm{~m} \times 64 \mathrm{~m}$. The correlative significance of species pairs changed from low to high, and if the quadrat size continued to increase, new relationships would emerge. These species pairs accounted for $29.4 \%$ of the total. For the forth type, the significant and very significant inter-specific associations changed from low to high and then from low to high again as the quadrat size continued to increase. These species pairs accounted for $11.8 \%$ of the total. Species
\end{abstract}


associations among shrub-herb and herb-herb pairs were more numerous in 17 inter-specific associations, accounting for $76.5 \%$ of the total. Species associations between Populus euphratica and arbor and shrub-shrub were distributed independently of each other. Even if inter-specific associations among species-pairs appeared, they were usually negative correlations. Most species-pairs, which were between arbor and herb-shrub and herb-herb, typically had positive correlations .

Key words Tarim River, Desert riparian forest community, Inter-specific relationship, Fisher's exact test for contingency table

种间关系是植物群落重要的数量和结构特征之 一。种间联接是指不同物种在空间分布的相互关联 性,通常是由于群落生境的差异影响了物种的分布 而引起的 (王伯荪和彭少麟, 1985; Greig-Smith, 1983)。测定不同种的种间联结 (Association)性大小 能揭示植物种在空间上共同出现或结合的程度。说 明植物种是独立分布, 还是存在相互吸引或排斥的 关系(宋永昌,2001)。可以深入了解群落结构、物种 间的相互作用、各种群与环境因子的关系。

近年来, 针对种间关系的研究方法和不同植被 类型的优势种种间的联结性, 国内学者作了不少工 作(王伯荪和彭少麟, 1985; 王祥荣和宋永昌, 1994; 杜道林等, 1995; 刘庆等, 1996; 孙伟中和赵士洞, 1997; 郭志华等, 1997; 李先琨等, 1999; 李新荣, 1999; 周先叶等, 2000; 郭相亿等, 2001; 张思玉, 2001; 汪建 华和李旭光, 2001)。也有少数学者对水生植物群落 和荒漠植物群落的种间关联性进行了研究 (孙学刚 等, 1998; 陈中义和陈家宽, 1999; 李正海和包雅静, 2000)。而对荒漠区生态环境脆弱带 (Ecotone) 中植 物群落种间关系的研究少有报道。种间联结的有效 性与取样面积有关, 但有关这方面研究不多, 且尚无 明确的定论 (彭少麟和王伯䔉, 1985; 陈中义和陈家 宽, 1999; 李德志等, 1996; 张峰和上官铁梁, 2000)。

本文旨在通过对新疆塔里木河中下游荒漠河岸 磷群落的种间关系分析, 研究不同取样面积对种间 联结的结果是否存在影响, 探讨是否存在最适的取 样面积, 了解荒漠河岸林群落内各种群之间的内在 联系。研究结果将对塔里木河流域受损植被恢复与 退耕还林还草后的植被重建有重要意义。

\section{1 研究区域概况}

研究区域位于新疆塔里木盆地北部塔里木河中下 游, 地处 $86^{\circ} 15^{\prime} \sim 88^{\circ} 00^{\prime} \mathrm{E}, 40^{\circ} 30^{\prime} \sim 41^{\circ} 30^{\prime} \mathrm{N}$, 属于暖温带 大陆荒漠气候。气候极端干旱, 年降水 $20 \sim 40 \mathrm{~mm}$, 蒸 发量 $2965 \mathrm{~mm}$ 。光照丰富, 年日照时数为 $3000 \mathrm{~h}$ 。年平 均温度高于 $10{ }^{\circ} \mathrm{C}$ 。最低气温 $-20{ }^{\circ} \mathrm{C}$, 最高气温 $39^{\circ} \mathrm{C}$ 。 该地区土壤为盐化草甸土、胡杨 (Populus euphratica) 林
土、盐土、风沙土和沼泽土等。本区植被具有温带荒漠 的特殊性,盆地植被呈紧缩型分布，在广大空间裸露而 于河流沿岸集结。分布较广的种类是在地表水条件极 为异质的生境中完成其生活周期的类型。由于受塔里 木河的影响, 非地带性的水分条件丰富了一些沿河岸 地貌分布占优势的沙漠植被类型, 出现了以胡杨为建 群种的荒漠河岸落叶阔叶林(杜加依林)。由于上游地 区补给干流水量年递减约 3000 万 $\mathrm{m}^{3}$, 导致中下游地下 水位下降, 自然植被日趋退化。近 10 年的盲目土地开 层活动,也使一部分天然植被受到破坏。

\section{2 研究方法}

\section{1 样带样方设置与调查方法}

2002 年 $7 \sim 9$ 月, 在新疆尉犁县城至新疆生产建 设兵团农二师 35 团范围内的塔里木河中下游荒漠 河岸林群落内, 设置了 3 条垂直于塔里木河河道的 样带。在样带内采用分层取样的方法对群落内不同 的植被层进行调查。在样带内以近似等距离设置 $30 \mathrm{~m} \times 30 \mathrm{~m}$ 的样地 21 个(图 1)。样地作为乔木样 方。沿每个样地对角线设 3 个 $10 \mathrm{~m} \times 10 \mathrm{~m}$ 的灌木 样方。沿每个样地的对角线作宽为 $2 \mathrm{~m}$ 的小样带 2 条, 每间隔 $2 \mathrm{~m}$ 设一个 $2 \mathrm{~m} \times 2 \mathrm{~m}$ 的草本样方。在不 同层次的样方中分别调查乔木、灌木和草本植物的 物种组成、株( 丛)高、冠(丛)幅和数量。

以 21 个样地为基点,运用巢式取样法(周纪伦 等, 1992), 分别设置了以 $2 \mathrm{~m} \times 2 \mathrm{~m}$ 为最小样方面 积, 依次向外以 2 倍面积递增的 10 种不同尺度 $(2 \mathrm{~m}$ $\times 2 \mathrm{~m}, 2 \mathrm{~m} \times 4 \mathrm{~m}, 4 \mathrm{~m} \times 4 \mathrm{~m}, 4 \mathrm{~m} \times 8 \mathrm{~m}, 8 \mathrm{~m} \times 8 \mathrm{~m}, 8 \mathrm{~m}$ $\times 16 \mathrm{~m}, 16 \mathrm{~m} \times 16 \mathrm{~m}, 16 \mathrm{~m} \times 32 \mathrm{~m}, 32 \mathrm{~m} \times 32 \mathrm{~m}$ 和 32 $\mathrm{m} \times 64 \mathrm{~m}$ ) 的样方, 共 210 个。分别记录不同级别样 方中出现的植物种类。植被调查的同时记录各样地 的环境要素。并在样地中心的空地挖土壤剖面, 采 集土样; 打观测井, 测地下水埋深位, 取水样。

\section{2 种间联结的测定}

种间联结的测定有联结指数 $I A$ ( Jaccard, 1901; Dice, 1945)、 $A C$ 和 $\chi^{2}$ 检验(张思玉, 2001; 郭志华等, 1997)等方法。对于样本数小于 40 或有单元格中的 


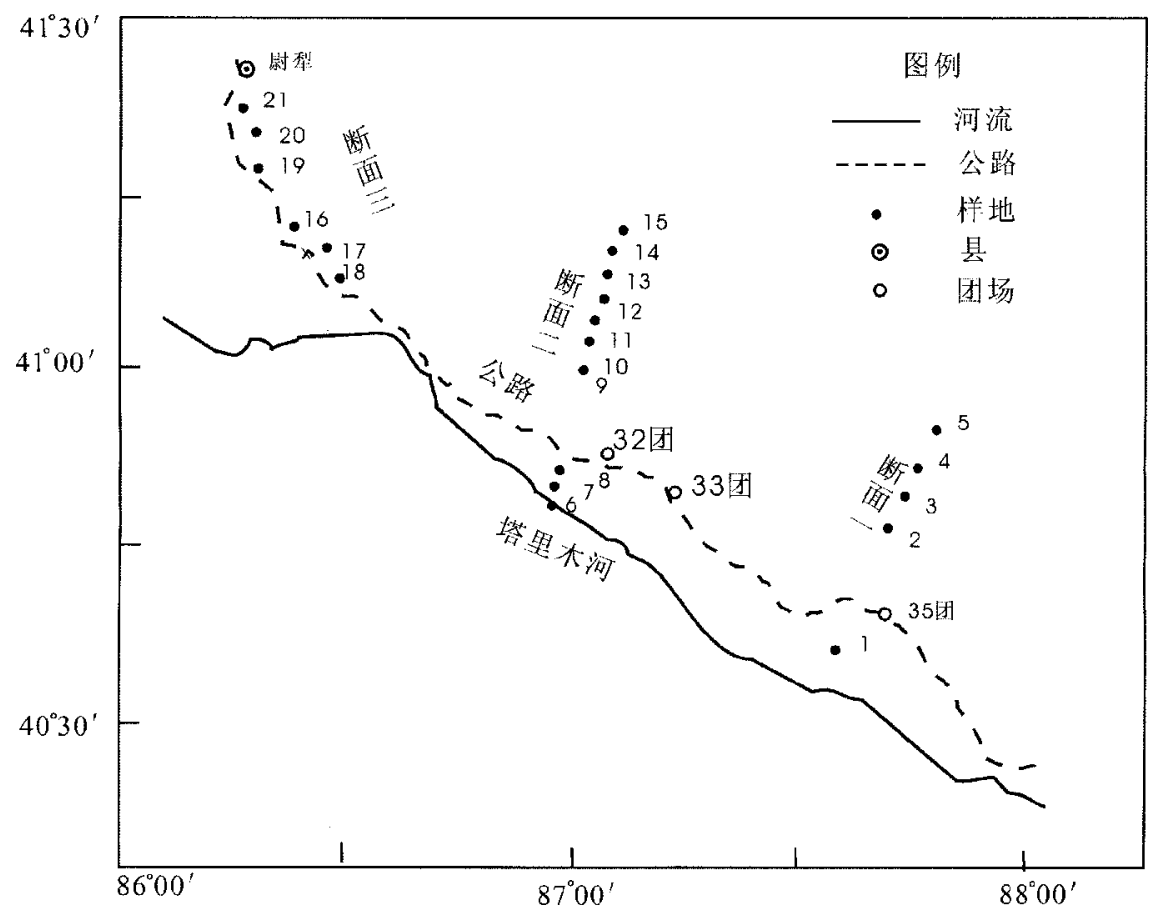

图 1 塔里木河中下游 3 条样带及 21 个样地的布设

Fig. 1 The distribution of 3 sectors and 21 sampling sites in the middle and lower reaches of Tarim River

期望频数小于 5 时, 适合使用 $2 \times 2$ 列联表的 Fisher 精确检验测定种间联结 (张峰和上官铁梁, 2000; 卢 纹岱, 2002)。在本研究中样方数为 21, 故采用 Fisher 精确检验法测定种间联结。

利用巢式取样法获取的定性数据, 按照不同的 样方面积尺度, 分别构建 $21 \times 16$ 的二元数据矩阵。 用 1 表示该物种存在, 0 表示该物种不存在。应用 SPSS10.0 软件进行 120 个种对的 $2 \times 2$ 联列表的 Fisher 精确检验（吴仲贤，1993）。任一联列表的 Fisher 精确检验表达式如下:

$$
p(1)=\frac{(a+b) !(c+d) !(a+c) !(b+d) !}{N ! a ! b ! c ! d !}
$$

对于任一 $2 \times 2$ 列联表, 精确检验首先按公式, 求出 $p(1)$ 。然后在保持行、列总数 $(a+b, c+d, a$ $+c, b+d)$ 不变的前提下, 将表中最小的值, 逐个降 低直到为 0 。每降低一次, 按照公式求 $p(2) 、 p(3)$ 、 $\cdots \cdots p(j) \cdots \cdots, p=\sum_{i=1}^{m} p(i)$ 即为其精确检验的结果。

\section{3 结果与分析}

\section{1 研究区的植物物种与结构组成}

荒漠河岸林植物种类少, 群落结构简单。在研 究区记录到的高等植物约有 30 种, 分别属于 13 科 28 属。 $1000 \mathrm{~m}^{2}$ 的范围内一般有植物 $3 \sim 8$ 种。在
水土条件较好的地段植物种类可达到 10 种以上。 环境条件极端恶劣的地段可见到仅有 $1 \sim 2$ 种植物 组成的群落。

在所调查的 3 条样带 21 个样地中共计出现了 16 种植物 (表 1 ), 分属于 11 科 15 属。组成了研究

表 1 新疆塔里木河中下游 21 个样地内出现的植物种 Table 1 List of plant species in 21 sampling sites in the middle and lower reaches of Tarim River, Xinjiang

\begin{tabular}{cl}
\hline $\begin{array}{c}\text { 编号 } \\
\text { Code }\end{array}$ & \multicolumn{1}{c}{$\begin{array}{c}\text { 种名 } \\
\text { Species }\end{array}$} \\
\hline 1 & 胡杨 Populus euphratica \\
2 & 多枝柽柳 Tamarix ramosissima \\
3 & 刚毛柽柳 Tamarix hispida \\
4 & 塔里木沙拐柊 Calligonum roborovskii \\
5 & 黑果枸杞 Lycium ruthenicum \\
6 & 铃铛刺 Halimodendron halodendron \\
7 & 盐穗木 Halostashys caspica \\
8 & 盐爪爪 Kalidium foliatum \\
9 & 花花柴 Karelinia caspica \\
10 & 疏叶骆驼刺 Alhagi sparsifolia \\
11 & 大叶白麻 Poacynum hendersonii \\
12 & 芦苇 Phragmites australis \\
13 & 河西菊 Hexinia polydichotoma \\
14 & 喀什牛皮消 Cynanchum kashgaricum \\
15 & 胀果甘草 Glycyrrhiza inflata \\
16 & 蓼子朴 Inula salsoloides \\
\hline
\end{tabular}


区荒漠河岸林群落的主体。群落垂直结构最多为 乔、灌、草 3 层。在调查的 21 个样地中, 具 3 层结构 的有 4 个, 具 2 层结构的有 10 个, 仅具 1 层结构的 有 7 个。植物水平分布稀疏, 表现为植株个体间和 种与种之间的水平距离大。乔木层仅由胡杨组成; 灌木层以铃铛刺 (Halimodendron halodendron)、塔里 木沙拐冭( Calligonum roborovskii)、多枝柽柳( Tamarix ramosissima)、刚毛柽柳 ( Tamarix hispida)、盐穗木 (Halostashys caspica)、黑果枸杞 (Lycium ruthenicum) 和盐爪爪 (Kalidium foliatum $)$ 为主; 草本层以花花柴 (Karelinia caspica)、疏叶骆驼刺 (Alhagi sparsifolia)、 大叶白麻 (Poacynum hendersonii)、芦苇 (Phragmites australis)、河西菊 (Hexinia polydichotoma)、喀什牛皮 消( Cynanchum kashgaricum)、胀果甘草 ( Glycyrrhiza inflata) 和苶子朴 (Inula salsoloides) 为主。16 种植物 在 10 种不同尺度的 210 个样方中组成了 120 个种 对。

\section{2 取样面积的有效性}

不同种群是在一定的空间范围内发生作用的, 超过这个范围，其间的直接相互作用必将逐渐消失。 借助样方大小的变动, 可以明确反映种间联结性在
空间上的变化。种间关联的测定结果受取样面积影 响, 当样方过小或过大都不能正确反映种间关联的 性质(陈中义和陈家宽, 1999)。

经实地调查和土样水样分析测定, 在小于 $32 \mathrm{~m}$ $\times 32 \mathrm{~m}$ 的样方尺度内,地形、地下水位、土壤类型、 盐分、土壤 $\mathrm{pH}$ 值、有机质含量等环境要素基本相对 一致。但当样方面积大于 $32 \mathrm{~m} \times 32 \mathrm{~m}$, 由于水、土、 盐的微地形的变化, 导致上述环境要素发生异化。 这时样方内出现的植物种, 实际上已经包括了适应 于不同水、盐等立地条件的种下生态型。在这种大 样方面积下进行种间联结分析, 将会产生与小样方 截然不同的结果。

如表 2 所示, 例如牛皮消-河西菊这一种对, 在 样方面积从 $2 \mathrm{~m} \times 2 \mathrm{~m}$ 一直到 $32 \mathrm{~m} \times 32 \mathrm{~m}$ (除 $4 \mathrm{~m} \times$ $8 \mathrm{~m}$ 外)的范围内, 分析结果显示这一种对有显著正 关联; 而在样方面积为 $32 \mathrm{~m} \times 64 \mathrm{~m}$ 时, 结果显示这 一种对无显著关联。如果仅以 $32 \mathrm{~m} \times 64 \mathrm{~m}$ 样方尺 度下的结果作为依据, 有可能将本来有显著关联的 种对认定为是独立分布的种对。

因此仅在某一种样方面积下研究物种的种间联 结性,得到的结果可能是片面的、不完全的。并不存

表 2 不同样方尺度下植物种对的 Fisher 精确检验显著性 $p$ 值

Table $2 p$ value of plant species pairs produced by Fisher's exact test at different quadrats sizes

\begin{tabular}{|c|c|c|c|c|c|c|c|c|c|c|}
\hline \multirow{3}{*}{$\begin{array}{l}\text { 物种对 } \\
\text { Species pairs }\end{array}$} & \multicolumn{10}{|c|}{$\begin{array}{c}\text { 显著关联与极显著关联种对的 } p \text { 值 } \\
p \text { value of species-pairs being significant or very significant }\end{array}$} \\
\hline & \multicolumn{10}{|c|}{ 样方尺度 Quadrats size $\left(\mathrm{m}^{2}\right)$} \\
\hline & $2 \times 2$ & $2 \times 4$ & $4 \times 4$ & $4 \times 8$ & $8 \times 8$ & $8 \times 16$ & $16 \times 16$ & $16 \times 32$ & $32 \times 32$ & $32 \times 64$ \\
\hline $5-13$ & 0.043 & & & & 0.040 & & & & & \\
\hline $5-14$ & 0.043 & & & & 0.040 & & & & & \\
\hline $13-14$ & 0.043 & 0.043 & 0.043 & & 0.004 & 0.004 & 0.012 & 0.012 & 0.012 & \\
\hline $11-13$ & & & & 0.024 & 0.024 & 0.024 & & & & \\
\hline $9-12$ & & & & & -0.037 & -0.037 & & & & \\
\hline $11-14$ & & & & & 0.024 & 0.024 & 0.024 & & & \\
\hline $5-12$ & & & & & 0.037 & & 0.029 & 0.029 & 0.029 & 0.019 \\
\hline $1-2$ & & & & & & -0.046 & -0.007 & -0.007 & & \\
\hline $1-12$ & & & & & & 0.037 & & & & \\
\hline $5-11$ & & & & & & 0.040 & 0.014 & 0.018 & 0.002 & 0.007 \\
\hline $2-7$ & & & & & & & -0.046 & & & -0.018 \\
\hline $11-15$ & & & & & & & 0.009 & 0 & 0 & 0 \\
\hline $14-15$ & & & & & & & 0.024 & 0.040 & 0.040 & \\
\hline $1-7$ & & & & & & & & -0.046 & & \\
\hline $5-15$ & & & & & & & & & 0.007 & 0.007 \\
\hline $6-9$ & & & & & & & & & 0.040 & 0.017 \\
\hline $13-15$ & & & & & & & & & & 0.045 \\
\hline
\end{tabular}

$p<0.05$ 植物种对显著关联, $p<0.01$ 植物种对极显著关联, 空白为 $p>0.05$ 植物种对无显著关联 $p<0.05$ species pairs being significant, $p$ $<0.01$ species pairs being very significant, blank means $p>0.05$ species-pairs being no significant 物种对列中的数字表示不同的植物种/每个数字相 对应的种名参见表 1 Figures in the species pairs list denote the plant species, the correspondences of figures and species see Table 1 
在对样地内所有物种均有效的最适样方面积。因此 在分析种间关联时应综合参考各种样方面积尺度的 分析结果。

宋永昌 (2001) 曾提出取样面积过小, 种对可能 多是负联结, 取样面积过大, 则可能多为正联结。陈 中义和陈家宽 (1999) 对长喙毛茛泽泻 (Ranalisma rostratum) 群落内种间关联的研究也有相同的结果。 在小样方面积时, 出现的均为正联结, 在中等样方面 积和大样方面积时, 正、负联结均有出现, 并且种对 的正负联结性也不因为样方面积的增大而发生改 变。在本文的研究结果中并未出现这种情况。荒漠 河岸林群落内种对间的正负联结与取样面积的大小 之间的关系有关还有待于进一步研究。

Fisher 精确检验法测定结果 (表 2)显示: 120 个 种对中共有 17 个种对分别在不同的样方尺度中表 现出显著或极显著的种间联结, 约占总数的 $14.2 \%$; 其中 13 个种对为正关联, 4 个种对为负关联。

不同种对表现出种间联结的最小样方尺度不 同。表明不同种群之间发生作用的空间范围是不同 的。黑果枸杞-河西菊、黑果枸杞-牛皮消和河西菊牛皮消这 3 个种对表现出种间正联结的最小样方面 积为 $2 \mathrm{~m} \times 2 \mathrm{~m}$ 。随着样方尺度的扩大, 也在部分样 方尺度下表现出显著的种间正联结。黑果枸杞-河 西菊、黑果枸杞-牛皮消在 $8 \mathrm{~m} \times 8 \mathrm{~m}$ 样方尺度下也 表现出显著的种间正联结。河西菊-牛皮消除了 $4 \mathrm{~m}$ $\times 8 \mathrm{~m} 、 32 \mathrm{~m} \times 64 \mathrm{~m}$ 这 2 个样方尺度外, 在其余的样 方尺度中均表现出显著的种间正联结。结合 CCA 物种排序的结果 (图 2), 可以看出: 黑果枸杞、河西 菊和牛皮消 3 个植物种均适应水分条件较好、盐分 较轻的生长环境, 并且植株的个体均不大。因此在 很小的样方尺度 $(2 \mathrm{~m} \times 2 \mathrm{~m})$ 中, 就能够将物种间的 联结显示出来。

大叶白麻-河西菊表现出种间正联结的最小样 方面积为 $4 \mathrm{~m} \times 8 \mathrm{~m}$ 。花花柴-芦苇、大叶白麻-牛皮 消、黑果枸杞-芦苇表现出种间联结的最小样方面积 为 $8 \mathrm{~m} \times 8 \mathrm{~m}$ 。胡杨-多枝柽柳、胡杨-芦苇、黑果枸杞 -大叶白麻表现出种间联结的最小样方面积为 $8 \mathrm{~m} \times$ $16 \mathrm{~m}$ 。多枝柽柳-盐穗木、大叶白麻-胀果甘草、牛皮 消-胀果甘草表现出种间联结的最小样方面积为 16 $\mathrm{m} \times 16 \mathrm{~m}$ 。胡杨-盐穗木表现出种间负联结的最小样 方面积为 $16 \mathrm{~m} \times 32 \mathrm{~m}$ 。黑果枸杞-胀果甘草、铃铛刺 一花花柴表现出种间正联结的最小样方面积为 $32 \mathrm{~m}$ $\times 32 \mathrm{~m}$ 。河西菊-胀果甘草表现出种间正联结的最 小样方面积为 $32 \mathrm{~m} \times 64 \mathrm{~m}$ 。种对表现出显著或极显



图 2 物种的 CCA 二维排序图(李涛等, 2003)

Fig.2 A two-dimensional scatter plot of CCA ordination for plant species ( Li et al., 2003)

1. 胡杨 Populus euphratica 2. 多枝柽柳 Tamarix ramosissima 3. 刚毛柽柳 Tamarix hispida 4. 塔里木沙拐本 Calligonum roborovskii 5. 黑果枸杞 Lycium ruthenicum 6. 铃铛刺 Halimodendron halodendron 7. 盐穗木 Halostashys caspica 8. 盐爪爪 Kalidium foliatum 9. 花花 柴 Karelinia caspica 10. 疏叶骆驼刺 Alhagi sparsifolia 11. 大叶白麻 Poacynum hendersonii 12. 芦苇Phragmites australis 13. 河西菊 Hexinia polydichotoma 14. 喀什牛皮消 Cynanchum kashgaricum 15. 胀果 甘草 Glycyrrhiza inflata 16. 蓼子朴 Inula salsoloides HW: 地下水位 Groundwater depth WSOLT: 地下水酸碱度 Groundwater pH S1SOLT: 剖面各层总盐平均值 The average contents of salt in soil profile horizons S1PH: 剖面各层酸碱度平均值 The average of $\mathrm{pH}$ in soil profile horizons

$\mathrm{S} 1 \mathrm{~N}$ : 剖面各层有机质平均值 The average contents of organic matter in soil profile horizons S2SOLT: 剖面各层总盐最大值 The contents of salt maximum in soil profile horizons S2PH: 剖面各层酸碱度最大值 The $\mathrm{pH}$ maximum in soil profile horizons

著种间联结的最小样方面积与植株的个体大小和两 物种在 CCA 排序图上的距离有关。植株的个体越 大, 两物种在排序图上的距离越远, 相应的种对出现 种间联结的最小样方面积也越大。

从 $4 \mathrm{~m} \times 8 \mathrm{~m}$ 的样方尺度以后的每一个样方尺 度下均有新的种对表现出显著或极显著的种间联 结。绝大部分具有种间联结的种对出现在 $8 \mathrm{~m} \times$ $8 \mathrm{~m}$ 样方尺度以上, 且种对数几乎没有变化, 趋近于 定值 7(表 2, 图 3)。但每个样方尺度下出现的具有 种间联结的植物种对发生了改变。

\section{3 种间联结性的空间变化类型}

由表 2 可以看出, 中等样方中出现的具有种间 联结的种对与大样方中出现的具有种间联结的种对 具有补充性。有的物种对的种间联结仅在中等样方 中表现出来,而有的要在大样方中才表现出种间联 结。各种对的种间联结性随样方尺度的变化而发生 变化。根据各种对的种间联结变化规律, 将各种对 




图 3 不同样方尺度下出现的具有种间联结的种对

Fig. 3 The number of species pairs at different quadrats size

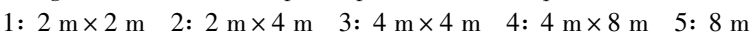
$\times 8 \mathrm{~m} \quad$ 6: $8 \mathrm{~m} \times 16 \mathrm{~m} \quad$ 7: $16 \mathrm{~m} \times 16 \mathrm{~m} \quad 8: 16 \mathrm{~m} \times 32 \mathrm{~m} \quad 9: 32 \mathrm{~m} \times 32$ m 10: $32 \mathrm{~m} \times 64 \mathrm{~m}$

归纳为 4 种类型 (表 3 )。

类型 I : 属于该类型的种对有 5-13、5-14、13-14。 这 3 个种对从最小样方尺度 $(2 \mathrm{~m} \times 2 \mathrm{~m})$ 到最大样方 尺度 $(32 \mathrm{~m} \times 64 \mathrm{~m})$, 种间的显著关联表现出从有到 无的循环变化。物种 5、13、14 在 CCA 物种排序图上 的位置很接近, 并且物种的个体也不大。所以通过 研究设定样方尺度能很充分地反映它们种间联结性 的变化。

类型 II : 包括的种对有 1-2、1-7、1-12、9-12、1113、11-14、14-15, 共计 7 个种对。在 10 个样方尺度 下, 这 7 个种对的种间显著关联表现出从无到有再 到无的变化。其中 3 个种对 1-2、1-7、9-12 表现为显 著或极显著的种间负关联。各种对的物种在 CCA 物种排序图上的距离较远。表明呈负关联的物种对 环境的适应条件相差较大。例如胡杨-盐穗木。通 过盐穗木 CCA 物种排序图上的位置, 反映它适应生 长在水分条件稍好, 但盐分较重的生境中。这与盐 穗木代表了典型的多汁盐柴类型, 对盐地生境具有 很强的占有能力的特征相一致。胡杨在 CCA 物种 排序图上的位置表明, 胡杨接近 CCA 物种排序轴的 中心。在 16 种物种中, 胡杨对水分、盐分和碱的耐 受能力适中。胡杨与盐穗木有较大的生境差异, 两 者表现出负关联。这些种对是在中等或较大样方尺 度 $(4 \mathrm{~m} \times 8 \mathrm{~m} \sim 16 \mathrm{~m} \times 32 \mathrm{~m}$ )下表现出种间关联。种 对 11-13、11-14、14-15 表现为种间正关联。物种 11、 13、14、15 对水分条件的需求比较接近, 盐分和碱分 相差较大。这些物种均为草本, 个体不大, 因此在较 大的样方尺度下表现出种间关联。

类型 III : 包括的种对有 5-11、5-15、6-9、11-15、1315 。这些显著或极显著的种间关联出现在 $8 \mathrm{~m} \times 16$ $\mathrm{m}$ 样方尺度以上, 直到最大样方尺度 $32 \mathrm{~m} \times 64 \mathrm{~m}$ 。 其中有 3 个种对为灌木与草本的种对, 2 个为草本 之间的种对。物种的个体不大。种间关联从无到 有, 可能随着取样尺度的扩大, 种间关联可能出现新 的变化。

类型 $\mathrm{IV}$ : 包括的种对有 2-7、5-12。随着样方尺 度的扩大, 显著或极显著的种间关联从无到有, 再从 无到有的循环变化。其中多枝柽柳与盐穗木为负关 联。多枝柽柳是荒漠地区的广泛分布的种类, 是典 型的荒漠-半荒漠河谷及泛滥地上的种类, 特别需要 含水量较多的土壤环境, 也能耐一定的盐, 在不同程 度的盐渍化土壤上都有分布。但它不是一个真盐生 植物。前人的研究也表明刚毛柽柳与盐穗木有较强 的负联结性, $A C$ 值为 -0.7 (孙学刚等, 1998), 与本 研究结果相似。

在 17 个具有种间联结的种对中, 属于类型 I 的 有 3 个种对, 占总数的 $17.6 \%$; 属于类型 II 的有 7 个 种对, 占总数的 $41.2 \%$; 属于类型 III 的有 5 个种对, 占总数的 $29.4 \%$, 属于类型IV 的有 2 个种对, 占总数 的 $11.8 \%$ 。植物种对关联与负关联的数量具有互 补性, 其合计数趋向一个恒数(表 3)。

在荒漠地区, 植物的生存环境恶劣。土壤养分 和水分条件差, 具有相同或相似生态位的种群与种 群之间的距离较大, 单位面积内植物多度和密度降 低。因此大部分种对在中等样方 $(8 \mathrm{~m} \times 8 \mathrm{~m} \sim 16 \mathrm{~m}$ $\times 16 \mathrm{~m})$ 和大样方 $(16 \mathrm{~m} \times 32 \mathrm{~m} \sim 32 \mathrm{~m} \times 32 \mathrm{~m})$ 面积下 才表现出种间联结性。植株个体较小, 并且适宜在 水分条件较好的环境下生长的草本植物种类, 如河 西菊与牛皮消。在处于在水分条件较好的局部环境 中, 同种植物个体间的距离以及不同种群之间的距 离相应较近, 因此从较小的样方面积到较大的样方 面积均可表现出不同程度的种间关联性。种间联结 性随空间变化的 4 种类型正是群落水平结构特征和 不同种对环境条件适应的的一种间接反映。

17 个具种间联结的种对以灌木-草本和草本-草 本的种对居多, 占了总数的 $76.5 \%$ (表 3 )。从表中 可以看出, 研究区主要乔木树种胡杨与灌木之间、灌 木和灌木之间是趋向独立分布的。如果在一定面积 的样方内出现有关联的种对, 也常常表现出负关联 的种间关系。这是因为这些相对高大的木本植物个 体有着较深较广的根系, 需要较大的空间来满足对 土壤水分的需求。乔木与草本植物之间, 灌木与草 本植物之间, 以及草本与草本之间, 由于处在不同的 生态位, 根系以及地上部分对光热和水分等资源的 
表 3 不同样方尺度下植物种对的关联显著性

Table 3 Correlative significance of plant species pairs in different quadrats sizes

\begin{tabular}{|c|c|c|c|c|c|c|c|c|c|c|c|}
\hline \multirow{2}{*}{$\begin{array}{l}\text { 类型 } \\
\text { Type }\end{array}$} & \multirow{2}{*}{$\begin{array}{c}\text { 物种对 } \\
\text { Species pairs }\end{array}$} & \multicolumn{10}{|c|}{ 样方尺度 Quadrats size $\left(\mathrm{m}^{2}\right)$} \\
\hline & & $2 \times 2$ & $2 \times 4$ & $4 \times 4$ & $4 \times 8$ & $8 \times 8$ & $8 \times 16$ & $16 \times 16$ & $16 \times 32$ & $32 \times 32$ & $32 \times 64$ \\
\hline \multirow[t]{3}{*}{ 类型 I Type I } & $5-13$ & * & & & & * & & & & & \\
\hline & $5-14$ & * & & & & * & & & & & \\
\hline & $13-14$ & * & * & * & & $* *$ & $* *$ & * & * & * & \\
\hline \multirow[t]{7}{*}{ 类型 II Type II } & $1-2$ & & & & & & $-*$ & $-* *$ & $-* *$ & & \\
\hline & $1-7$ & & & & & & & & $-*$ & & \\
\hline & $1-12$ & & & & & & * & & & & \\
\hline & $9-12$ & & & & & $-*$ & $-*$ & & & & \\
\hline & $11-13$ & & & & * & * & * & & & & \\
\hline & $11-14$ & & & & & * & * & * & & & \\
\hline & $14-15$ & & & & & & & * & * & * & \\
\hline \multirow[t]{5}{*}{ 类型 III TypeIII } & $5-11$ & & & & & & * & * & * & * * & $* *$ \\
\hline & $5-15$ & & & & & & & & & ** & $* *$ \\
\hline & $6-9$ & & & & & & & & & * & * \\
\hline & $11-15$ & & & & & & & ** & * * & ** & $* *$ \\
\hline & $13-15$ & & & & & & & & & & * \\
\hline \multirow[t]{2}{*}{ 类型IV TypeIV } & $2-7$ & & & & & & & $-*$ & & & $-*$ \\
\hline & $5-12$ & & & & & * & & * & * & * & * \\
\hline \multicolumn{2}{|c|}{ 正关联种对数 } & & & & & & & & & & \\
\hline \multicolumn{2}{|c|}{$\begin{array}{c}\text { Number of species pairs being } \\
\text { positively correlative } \\
\text { 负关联种对数 }\end{array}$} & 3 & 1 & 1 & 1 & 6 & 5 & 6 & 5 & 7 & 6 \\
\hline \multicolumn{2}{|c|}{$\begin{array}{c}\text { Number of species pairs being } \\
\text { negatively correlative }\end{array}$} & 0 & 0 & 0 & 0 & 1 & 2 & 2 & 2 & 0 & 1 \\
\hline \multicolumn{2}{|c|}{ 总计 Total } & 3 & 1 & 1 & 1 & 7 & 7 & 8 & 7 & 7 & 7 \\
\hline
\end{tabular}

* : 显著关联 Significant ** : 极显著关联 Very significant 物种对列中的数字表示不同的植物种, 每个数字相对应的种名同表 1 Figures in the species pairs list denote the plant species, the correspondences of figures and species see Table 1

表 44 种类型的种对统计

Table 4 The species-pairs statistics of 4 types

\begin{tabular}{|c|c|c|c|c|c|c|}
\hline & $\begin{array}{c}\text { 乔木与灌木 } \\
\text { Wood and shrub }\end{array}$ & $\begin{array}{c}\text { 乔木与草本 } \\
\text { Wood and herb }\end{array}$ & $\begin{array}{c}\text { 灌木与灌木 } \\
\text { Shrub layer }\end{array}$ & $\begin{array}{c}\text { 灌木与草本 } \\
\text { Shrub and herb }\end{array}$ & $\begin{array}{c}\text { 草本与草本 } \\
\text { Herb layer }\end{array}$ & $\begin{array}{l}\text { 合计 } \\
\text { Total }\end{array}$ \\
\hline 类型 I Type I & 0 & 0 & 0 & 2 & 1 & 3 \\
\hline 类型 II Type II & 2 & 1 & 0 & 0 & 4 & 7 \\
\hline 类型 III Type III & 0 & 0 & 0 & 3 & 2 & 5 \\
\hline 类型 IV TypeIV & 0 & 0 & 1 & 1 & 0 & 2 \\
\hline 合计 Total & 2 & 1 & 1 & 6 & 7 & 17 \\
\hline$\%$ & 11.7 & 5.9 & 5.9 & 35.3 & 41.2 & 100 \\
\hline
\end{tabular}

获取未产生大的冲突, 往往能形成较稳定的种间关 系。这些不同生活型植物所构成的种对多数有着一 定的正关联性。

以往的研究表明, 种间出现正联结的原因主要 有: 1)相同或相似的生境适应范围;2)物种间存在依 赖关系,一种依赖于另一种或两种相互依赖。种间 出现负联结的原因主要是: 1) 不相似的环境适应范 围;2)空间上的排斥或资源上的竞争;3)化学互感的 作用 (Greig-Smith, 1983; Kershw \& Looney, 1985; 宋永 昌, 2001)。而塔里木河中下游荒漠河岸林群落中种 间正关联的形成主要是一部分植物种具有相同或相
似的生态适应性,彼此间处于不同的生态位; 而另一 些植物种在一定的土壤水平和垂直空间范围内和在 一定的时期内对水资源的需求产生了排斥性竞争。

\section{4 小 结}

21 个样地中纪录到的 16 种植物组成了荒漠河 岸林群落的主体。群落垂直结构最多为乔、灌、草 3 层。具 3 层结构的有 4 个, 具 2 层结构的有 10 个, 仅具 1 层结构的有 7 个。 16 种植物在 10 种不同面 积尺度的 210 个样方中组成了 120 个种对。其中有 17 个种对表现出显著或极显著的种间联结, 约占总 
数的 $14.2 \% ; 13$ 个种对为正关联, 4 个种对为负关 联。塔里木河中下游荒漠河岸林群落中种间正关联 的形成主要是一部分植物种具有相同或相似的生态 适应性, 彼此间处于不同的生态位; 而另一些植物种 在一定的土壤水平和垂直空间范围内和在一定的时 期内对水资源的需求产生了排斥性竞争。

取样面积对种间联结性测定的有效性有影响。 不存在对样地内所有物种均有效的最适样方面积。 塔里木河荒漠河岸林群落的种间联结性分析适宜在 $8 \mathrm{~m} \times 8 \mathrm{~m} \sim 30 \mathrm{~m} \times 30 \mathrm{~m}$ 样方面积尺度内进行, 其结 果有效性强。不同种对表现出种间联结的最小样方 尺度不同, 荒漠河岸林不同种群之间发生作用的空 间范围存在着差异。

随着样方面积的增大, 种间联结有各自不同的 变化规律。据此概括为 4 种类型: 类型 $I$ : 种对从最 小样方尺度 $(2 \mathrm{~m} \times 2 \mathrm{~m}$ ) 到最大样方尺度 $(32 \mathrm{~m} \times 64$ $\mathrm{m})$, 种间的显著关联表现出从有到无的循环变化。 这类种对占总数的 $17.6 \%$; 类型 II : 种对的种间显 著关联随着样方面积的增大, 表现出从无到有再到 无的变化。这类种对占总数的 $41.2 \%$; 类型 III : 显 著或极显著的种间关联出现在 $8 \mathrm{~m} \times 16 \mathrm{~m} \sim 32 \mathrm{~m} \times$ $64 \mathrm{~m}$ 的范围内。种间关联从无到有, 可能随着取样 尺度的继续扩大, 种间关联会出现新的变化。这类 种对占总数的 $29.4 \%$; 类型 $\mathrm{IV}$ : 随着样方尺度的扩 大, 显著或极显著的种间关联从无到有, 再从无到有 的循环变化。属于此类型种对占总数的 $11.8 \%$ 。

17 个具种间联结的种对以灌木-草本和草本-草 本的种对居多, 占了总数的 $76.5 \%$ 乔木树种胡杨与 灌木之间、灌木和灌木之间是独立分布的。既使出 现有关联的种对，也常常表现出负关联的种间关系。 乔木与草本植物之间, 灌木与草本植物之间, 以及草 本与草本之间,所构成的种对多数有着一定的正关 联性。

\section{参 考 文 献}

Chen ZY (陈中义), Chen JK (陈家宽) (1999). The spatial pattern of Ranaliama rostratum population and interspecific association in the community. Acta Phytoecologica Sinica (植物生态学 报), 23, 56-61. (in Chinese with English abstract)

Dice LR (1945). Measures of the amount of ecologic association between two species. Ecology, 26, 297 - 302 .

Du DL (杜道林), Liu YC (刘玉成), Li R (李睿) (1995). Studies on the interspecific association of dominant species in a subtropical Castanopsis fargesii forest of Jinyun Mountain, China. Acta Phytoecologica Sinica (植物生态学报), 19, 149-157. (in Chinese with English abstract)

Greig-Smith P (1983) . Quantitative Plant Ecology 3rd edn. Black- well Scientific Publications, Oxford, $105-128$.

Guo XY (郭相亿), Li YH (李裕红), Lin HP (林慧萍) (2001). Studies on the inter-specific association among main species of Cyclocarya paliurus community in Niumulin Natural Reserve. Journal of Fujian College of Forestry (福建林学院学 报), 21, 181-185. (in Chinese with English abstract)

Guo ZH (郭志华), Zhuo ZD (卓正大), Chen J (陈洁), Wu MF (吴梅风) (1997). Interspecifica association of trees in mixed evergreen and deciduous broadleaved forest in Lushan Mountain. Acta Phytoecologica Sinica (植物生态学报), 21, 424 - 432. (in Chinese with English abstract)

Jaccard P (1901). Etude comparative de al distribution florale dans une portion des Alpes et du Jura. Bulletin de la Société Vaudoise des Sciences Naturelles, 37,547-597.

Kershaw KA, Looney JH (1985). Quantitative and Dynamic Plant Ecology 3rd edn. Edward Arnold Limitid, London, 78 - 94.

Li DZ (李德志), Qin AL (秦艾丽), Yang ML (杨茂林), Liu PF (刘鹏飞) (1996). Study on the interconnections among main tree populations in natural secondary forest commuities. Acta Phytoecologica Sinica (植物生态学报), 20, 263-271. (in Chinese with English abstract)

Li T (李涛), Yin LK (尹林克), Yan C (严成) (2003). Quantitative classification and ordination analysis on vegetation in the middle reaches of Tarim River. Arid Land Geography (干旱区 地理), 26, 173 - 179. (in Chinese with English abstract)

Li XK (李先琨), Huang YQ (黄玉清), Su ZM (苏宗明) (1999). Preliminary studies on interconnections among the main tree populations of Taxus chinensis var. mairei co mmunity. Chinese Journal of Ecology (生态学杂志), 18, 10 - 14. (in Chinese with English abstract)

Li XR (李新荣) (1999). Interspecific association and correlation of shrub layer in the coniferous-broad leaved mixed geobotanical zone of Russia plain. Acta Ecologica Sinica (生态学报), 19, 55 - 60. (in Chinese with English abstract)

Li ZH (李正海), Bao YJ (包雅静) (2000). Study on Changes of population pattern and interspecific relationship of Caragana in Inner Mongolia Steppe and desert region. Journal of Arid Land Resources and Environment (干旱区资源与环境), 14, 64 68. (in Chinese with English abstract)

Liu Q (刘庆), Bao WK (包维楷), Qiao YK (乔永康), Qian NB (钱能斌) (1996). Studies on the interspecific relationship among dominant species of the semi-arid valley scrubs in Moxian on the upper reaches of the Minjiang River. Chinese Journal of Applied Environmental Biology (应用与环境生物学报), 2, 36 - 42. (in Chinese with English abstract)

Lu WD (卢纹岱) (2002). SPSS for Windows (社会科学统计程 序). Electronic Industry Press, Beijing, 115. (in Chinese)

Peng SL (彭少麟)，Wang BS (王伯称) (1985). The measured technique of interspecific association of the lower subtropical evergreen-broadleaved forest. II . The sample technique. Tropical and Subtropical Forest Ecosystem (热带亚热带森林生态系统研 究) , 3, $167-173$. (in Chinese with English abstract)

Song YC (宋永昌) (2001). Vegetation Ecology (植被生态学). East China Normal University Press, Shanghai, 353 - 417. (in Chinese)

Sun XG (孙学刚), Xiao W (肖雯), Jia HX (贾恢先) (1998). Invertigation on the community structure, spatial pattern of popu- 
lation and interspecific association of Tamarix hispida saline desert in the middle reaches of Shule River. Acta Prataculturae Sinica (草业学报), 7 (2), 10 - 17. (in Chinese with English abstract)

Sun WZ (孙伟中), Zhao SD (赵世洞) (1997). Distribution patterns of main tree species in Titia broadleaf Korean pine forest on northern slope of Changbai Mountains. Chinese Journal of Applied Ecology (应用生态学报), 7, 1-5. (in Chinese with English abstract)

Wang BS (王伯荪), Peng SL (彭少麟) (1985). Studies on the measuring techniques of interspecific association of the lower subtropical evergreen-broadleaved forest. I. The exploration and the revision on the measuring formulas of interspecific assocoation. Acta Phytoecologica et Geobotanica Sinica (植物生态学与地植 物学丛刊), 9, 274-285. (in Chinese with English abstract) Wang JH (汪建华), Li XG (李旭光) (2001). Study on the interconnections among main tree population in the evergreen broadleaf forest in $\mathrm{Si}$ mian Mountain, Chongqing. Journal of Yuzhou University (Natural Science Edition) (渝州大学学报 (自然科学版) ), 18 (3), 58-62. (in Chinese with English abstract)

Wang XR (王祥荣), Song YC (宋永昌) (1994). Interspecific correlation of evergreen broad-leaved in Tiantong National Forest Park of Zhejiang Province. Chinese Journal of Ecology (应用生 态学报), 5, 113 - 119. (in Chinese with English abstract)

Wu ZX (吴仲贤) (1993).Biostatistics (生物统计学). Beijing Agricultural University Press, Beijing, 393 - 395. (in Chinese)

Zhang F (张峰), Shangguan TL (上官铁梁) (2000). Numerical analysis of interspecific relationships in an Elaeagnus molls community in Shanxi. Acta Phytoecologica Sinica (植物生态学报), 24, 351 - 355. (in Chinese with English abstract)

Zhang SY (张思玉) (2001). Interspecific association of main tree populations in Alsophila spinulosa community. Chinese Journal of Applied Environmental Biology (应用与环境生物学报), 7, 335 - 339. (in Chinese with English abstract)

Zhou XY (周先叶), Wang BS (王伯荪), LI MG (李鸣光), Zan QJ (处启杰) (2000). An analysis of interspecific associations in secondary succession forest communities in Heishiding Natural Reserve, Guangdong Privince. Acta Phytoecologica Sinica (植物生态学报), 24, 332 - 339. (in Chinese with English abstract)

Zhou JL (周纪伦), Zheng SZ (郑师章), Yang C (扬持) (1992). Plant Population Ecology (植物种群生态学). Higher Education Press, Beijing, 96-115, 215 - 235. (in Chinese) 